- ORIGINAL ARTICLE Volume 8 Issue 42016

DOI: 10.5959/eimj.v8i4. 473

ARTICLE INFO

Submitted: $10-10-2016$

Accepted: 13-11-2016

Online: $30-12-2016$

\section{Knowledge of Blood Transfusion among Nurses at Hospital Pulau Pinang: Nursing Responsibilities and Patient Management Related to Transfusion Reactions}

\author{
Elaine Lim Siew Lee, Nur Arzuar Abdul Rahim, Sharifah Azdiana \\ Tuan Din \\ Cluster of Regenerative Medicine, Advanced Medical and Dental \\ Institute, Universiti Sains Malaysia, Bertam, Pulau Pinang, \\ Malaysia
}

\begin{abstract}
To cite this article: Lim SL Elaine, Abdul Rahim NA, Tuan Din SA. 2016. Knowledge of blood transfusion among nurses at Hospital Pulau Pinang: nursing responsibilities and patient management related to transfusion reactions. Education in Medicine Journal. 8(4):47-56. DOI: 10.5959/eimj.v8i4.473
\end{abstract}

To link to this article: http://dx.doi.org/10.5959/eimj.v8i4.473

\begin{abstract}
Introduction: Nurses are generally trained on the procedures of blood transfusion during their years of service. However, the level of knowledge varies among individuals and healthcare centres. Thus, this study addresses a knowledge gap regarding nurses' knowledge and transfusion safety in the local clinical setting. Objectives: This study aimed to determine nurses' level of knowledge of various phases of blood transfusion and associate this knowledge with their socio-professional details. Methods: This was a cross-sectional, quantitative study. The target population was in-patient ward nurses at Hospital Pulau Pinang. The systematic random sampling method was used to select 185 nurses to participate in this study. Data were collected using a validated research questionnaire that consisted of 31 items (9 items related to socio-professional factors and 22 knowledge items). Data were analysed using descriptive and inferential analyses. Results: The overall knowledge score was moderate (mean $=70.44 \%, \mathrm{SD}=11.35)$. None of the nurses was able to answer all of the knowledge questions correctly. Of the participants, $72.29 \%, 71.75 \%$, and $67.14 \%$ exhibited knowledge of blood bag collection and patient preparation; pre-transfusion nursing activities; and during and post-transfusion nursing responsibilities and management of transfusion reactions, respectively. Factors such as age, years of service, department, and availability of the transfusion policy in the ward significantly affected the mean knowledge scores. Conclusion: Nurses' knowledge of blood transfusion at Hospital Pulau Pinang was moderate. More training and courses should be provided to improve their knowledge.
\end{abstract}

Keywords: Knowledge, Blood transfusion, Patient management, Transfusion reaction

CORRESPONDING AUTHOR Dr. Sharifah Azdiana Tuan Din, Cluster of Regenerative Medicine, Advanced Medical and Dental Institute, Universiti Sains Malaysia, Bertam, 13200 Kepala Batas, Pulau Pinang, Malaysia | Email: azdiana@usm.my

\section{INTRODUCTION}

Since its establishment in the early twentieth century, blood transfusion, whereby blood or its constituents are infused to individuals through intravenous administration, has been one of the most common procedures administered to hospitalised individuals (1). The transfusion of blood products is essential for restoring the body's oxygen transport capacity or replenishing lost or depleted blood components (2). However, blood transfusion has certain risks to recipients, including transmission 
of transfusion-transmitted infections, acute or delayed transfusion reactions, alloimmunisation and immunomodulation (3). Extensive research and efforts have been focused on preventing these undesired events.

Errors in blood transfusion can lead to severe morbidity or even mortality of recipients (4). Serious Hazards of Transfusion reported that approximately one wrong blood transfusion occurred in every 13,000 transfusions (5). Most transfusion errors are due to human factors, which are preventable through training and revision of transfusion protocols (6). Hence, healthcare workers who play a part in blood transfusion service must always be competent and cautious to do no harm and to provide safe and beneficial transfusion therapy to the patients.

Hijji et al. described administration of blood products as consisting of five phasic procedures: decision to transfuse, patient preparation before collecting blood units from the storage site, blood bag collection, pre-transfusion activities, and posttransfusion activities and monitoring (7). While decisions to transfuse are determined by physicians, other phases are controlled by the operators and nursing staff (8). All phases need to be monitored and safeguarded by multiple parties to ensure efficacy and efficiency of the transfusion. Safe blood administration must be accompanied by proper documentation of related tasks, especially of reasons, time, duration, and operators, to ensure traceability and to facilitate look back procedures (4).

Nurses are generally trained on the procedures of blood transfusion during their years of service. However, the level of knowledge varies among individuals and healthcare centres. Although studies to evaluate nurses' knowledge of blood transfusion have been conducted in other countries (7-12), few have taken place in Malaysia. Thus, this study addresses a knowledge gap regarding nurses' knowledge and transfusion safety in the local clinical setting. Nurses' knowledge at various stages of blood transfusion was assessed and the data were used to identify areas in need of improvement. Policy makers can use the results of this study to make adjustments and outline strategies to improve the quality of blood transfusion service in their healthcare facilities.

\section{METHODS}

\section{Design and Sample}

This was a cross-sectional, quantitative study conducted at Hospital Pulau Pinang, Malaysia. The target population was registered nurses based in in-patient wards with at least three months of experience in adult blood transfusion prior to the data collection period. Paediatric and neonatal ward nurses were excluded from this study. With reference to Hijji et al., sample size was calculated using the Power and Sample Size Program (version 3.0) (7). Assuming a 95\% confidence interval and power of the study at $80 \%$, the minimum sample size was 185 nurses. Nurses from a total of 23 wards, including the accident and emergency unit, cardiology ward, critical care unit, intensive care unit (ICU), medical wards, neurologyICU, neurology-surgical ward, obstetrics and gynaecology ward, orthopaedic wards, and surgical wards, participated in this study.

\section{Ethical Consideration}

This study was approved by the Human Research Ethics Committee at Universiti Sains Malaysia and the Medical Research and Ethics Committee. Approval to carry out this research at the study location was obtained from the hospital director, head of nursing, and Clinical Research Centre. Every subject was given an information sheet and was required to sign an informed consent form prior to participation. Anonymity and confidentiality of responses were guaranteed. 


\section{Research Tool}

Following a thorough literature review on blood transfusion and nursing responsibilities including research articles and nursing guidelines, the questionnaire was designed, based on the study by Hijji et al. (8). The adopted questions were modified in correspondence to the local clinical policies and settings.

The questionnaire consisted of four sections containing a total of 31 items: Section 1 consisted of nine items regarding "socioprofessional factors"; Section 2 contained seven items related to "blood bag collection from blood bank and patient preparation before transfusion"; Section 3 was made up of eight items regarding "pre-transfusion nursing responsibilities"; Section 4 had seven items related to "during and posttransfusion nursing responsibilities and management of adverse reactions". The research tool was tested previously for its content validity, face validity, and reliability.

\section{Data Collection}

Data were collected from May to June 2016. Subject selection was conducted via the systematic random sampling method as follows: nurses whose names were listed with odd numbers on the ward duty rosters were included, whereas even-numbered names were excluded. A briefing session with fellow matrons from every participating in-patient ward was held. Standardised verbal and written instructions were given to the matrons before they distributed the questionnaire to the selected nurses.

\section{Data Analysis}

Data entry and analysis were done using the statistical software Statistical Package for Social Sciences (SPSS, version 22.0). The data were analysed using descriptive and inferential analyses. Descriptive analysis was used to analyse the socio- professional factors and individual items in each section. For Sections 2-4, one point was assigned to a correct response while no point was given to an incorrect answer. The knowledge items summed to a total score of 22, and correct answers were converted to a percentage for easy interpretation. Measures of central tendency and dispersion were calculated from the knowledge score. Inferential statistics were used to analyse the association between each socio-professional factor and the knowledge score. To analyse relationships between categorical variables and knowledge score, the independent $t$-test was used for dichotomous variable, whereas one-way analysis of variance (ANOVA) was used if variable had more than two categories. Pearson's correlation coefficient was used for continuous data.

\section{RESULTS}

\section{Subjects' Socio-Professional Characteristics}

A total of 185 nurses participated in this study, and $96.2 \%$ of participants were female. The mean age of subjects was 30.93 years old $(\mathrm{SD}=7.49$; range $=23$ to 59). Sixty percent $(60 \%)$ of nurses were between 21 to 30 years old. The mean years of service was 7.41 . Years of service ranged from 8 months to 23 years (mean $=7.41, \mathrm{SD}=7.06$ ) years. Most of the nurses $(93.5 \%)$ had a basic qualification in nursing (either a nursing certificate or diploma). Half of the nurses stated that they performed blood transfusion only once a month. Participating wards were further divided into 11 different departments. Nurses from the medical and surgical wards, where blood transfusion is actively performed, constituted more than one-third of the total sample. Eighty-five percent of nurses were aware that the policy related to administration of blood is available to them in their respective ward, whereas $\sim 12 \%$ were unsure (refer Table 1). 
Table 1: Socio-professional details and association with knowledge scores

\begin{tabular}{|c|c|c|c|}
\hline Socio-professional details & $n(\%)$ & Mean knowledge score \pm SD & Test statistics \\
\hline \multicolumn{4}{|l|}{ Gender $(n=185)$} \\
\hline Female & $178(96.2)$ & $70.76 \pm 11.14$ & $t(183)=1.94$ \\
\hline Male & $7(3.8)$ & $62.34 \pm 14.54$ & $p=0.054$ \\
\hline \multicolumn{4}{|c|}{$\begin{array}{l}\text { Age groups }(n=185) \\
\left(\operatorname{mean} \pm S D=30.93 \pm 7.49, r=0.194, p=0.008^{*}\right)\end{array}$} \\
\hline $21-25$ years & $39(21.1)$ & $65.97 \pm 13.31$ & $F(7,177)=3.118$ \\
\hline $26-30$ years & $72(38.9)$ & $69.07 \pm 9.82$ & $n=0004^{*}$ \\
\hline $31-35$ years & $41(22.2)$ & $74.94 \pm 10.02$ & \\
\hline $36-40$ years & $17(9.2)$ & $72.99 \pm 10.84$ & \\
\hline $41-45$ years & $3(1.6)$ & $75.76 \pm 10.50$ & \\
\hline $46-50$ years & $3(1.6)$ & $74.24 \pm 18.37$ & \\
\hline $51-55$ years & $7(3.8)$ & $77.27 \pm 10.50$ & \\
\hline $56-60$ years & $3(1.6)$ & $60.61 \pm 6.94$ & \\
\hline \multicolumn{4}{|c|}{$\begin{array}{l}\text { Years of service }(n=182) \\
\left(\text { mean } \pm S D=7.41 \pm 7.06, r=0.211, p=0.004^{*}\right)\end{array}$} \\
\hline$<1$ year & $2(1.1)$ & $59.09 \pm 6.43$ & $F(4,177)=7.332$ \\
\hline $1-3$ years & $53(29.1)$ & $67.24 \pm 10.89$ & $p<0.001^{*}$ \\
\hline $3-5$ years & $41(22.5)$ & $66.19 \pm 11.77$ & \\
\hline $5-10$ years & $48(26.4)$ & $74.91 \pm 9.65$ & \\
\hline$>10$ years & $38(20.9)$ & $75.12 \pm 10.34$ & \\
\hline \multicolumn{4}{|l|}{ Education level ( $n=185)$} \\
\hline Basic & $173(93.5)$ & $70.15 \pm 11.41$ & $t(183)=-1.321$ \\
\hline Advanced & $12(6.5)$ & $74.62 \pm 9.97$ & $p=0.188$ \\
\hline \multicolumn{4}{|c|}{ Frequency of transfusion $(n=184)$} \\
\hline Daily & $13(7.1)$ & $75.17 \pm 11.97$ & $F(5,178)=0.786$ \\
\hline Weekly & $35(19.0)$ & $70.78 \pm 12.04$ & $n=0561$ \\
\hline Fortnightly & $21(11.4)$ & $67.75 \pm 13.40$ & \\
\hline Monthly & $92(50.0)$ & $70.36 \pm 10.25$ & \\
\hline Yearly & $16(8.7)$ & $70.17 \pm 11.73$ & \\
\hline Never & $7(3.8)$ & $67.53 \pm 13.49$ & \\
\hline \multicolumn{4}{|l|}{ Departments $(n=185)$} \\
\hline$A \& E$ & $7(3.8)$ & $78.57 \pm 10.07$ & $F(10,47.786)=1.659$ \\
\hline $\mathrm{CCU}$ & $9(4.9)$ & $69.70 \pm 13.44$ & $p=0.119$ \\
\hline Cardiology & $15(8.1)$ & $69.39 \pm 14.32$ & \\
\hline ENT & $9(4.9)$ & $73.23 \pm 13.53$ & \\
\hline Haematology & $8(4.3)$ & $76.70 \pm 6.63$ & \\
\hline ICU & $19(10.3)$ & $72.01 \pm 15.84$ & \\
\hline Medical & $37(20.0)$ & $70.27 \pm 10.53$ & \\
\hline O\&G & $18(9.7)$ & $72.22 \pm 11.76$ & \\
\hline Orthopaedic & $13(7.0)$ & $65.38 \pm 11.52$ & \\
\hline Respiratory & $16(8.6)$ & $68.75 \pm 9.22$ & \\
\hline Surgical & $34(18.4)$ & $68.32 \pm 7.38$ & \\
\hline
\end{tabular}


Table 1: (continued)

\begin{tabular}{cccc}
\hline Socio-professional details & \multicolumn{1}{c}{$\boldsymbol{n}(\%)$} & Mean knowledge score $\mathbf{\pm}$ SD & Test statistics \\
\hline Availability of policy $(\boldsymbol{n}=\mathbf{1 8 3})$ & & & \\
Yes & $157(85.8)$ & $71.28 \pm 10.93$ & $\mathrm{~F}(2,180)=3.491$ \\
No & $5(2.7)$ & $66.36 \pm 13.48$ & $p=0.033^{*}$ \\
Not sure & $21(11.5)$ & $64.72 \pm 12.93$ & \\
\hline
\end{tabular}

${ }^{*} p<0.05$, statistically significant.

A\&E: accident and emergency; CCU: critical care unit; ENT: ear, nose, and throat; ICU: intensive care unit; O\&G: obstetrics and gynaecology

\section{Overall Knowledge Score}

Figure 1 summarises the frequency distribution of the knowledge score, which was normally distributed with a slight negative skew. The total knowledge score was 22 , and results were scaled to percentage. The mean overall knowledge score was $70.44 \% \quad(\mathrm{SD}=11.35)$, and the range was wide $(31.82 \%$ to $95.45 \%)$. Almost $80 \%$ of nurses obtained a score ranging from $50 \%$ to $80 \%$, with the highest frequency seen in the $70 \%$ to $79.99 \%$ range $(n=68)$.

\section{Knowledge of Blood Bag Collection from the Blood Bank and Patient Preparation before Transfusion}

The mean score in this section was $72.29 \%$ $(\mathrm{SD}=15.14$; range $=14.29$ to $100 \%)$. Over $90 \%(n=171)$ of subjects were able to answer more than half of the questions correctly. Of those participants, 11 answered all questions in this section correctly. Notably, almost $80 \%$ of nurses were unaware of the valid duration of the Group and Cross Match (GXM) sample. Most subjects $(n=101)$ had poor knowledge about $\mathrm{ABO}$ and $\mathrm{RhD}$ blood group compatibility (refer Table 2).

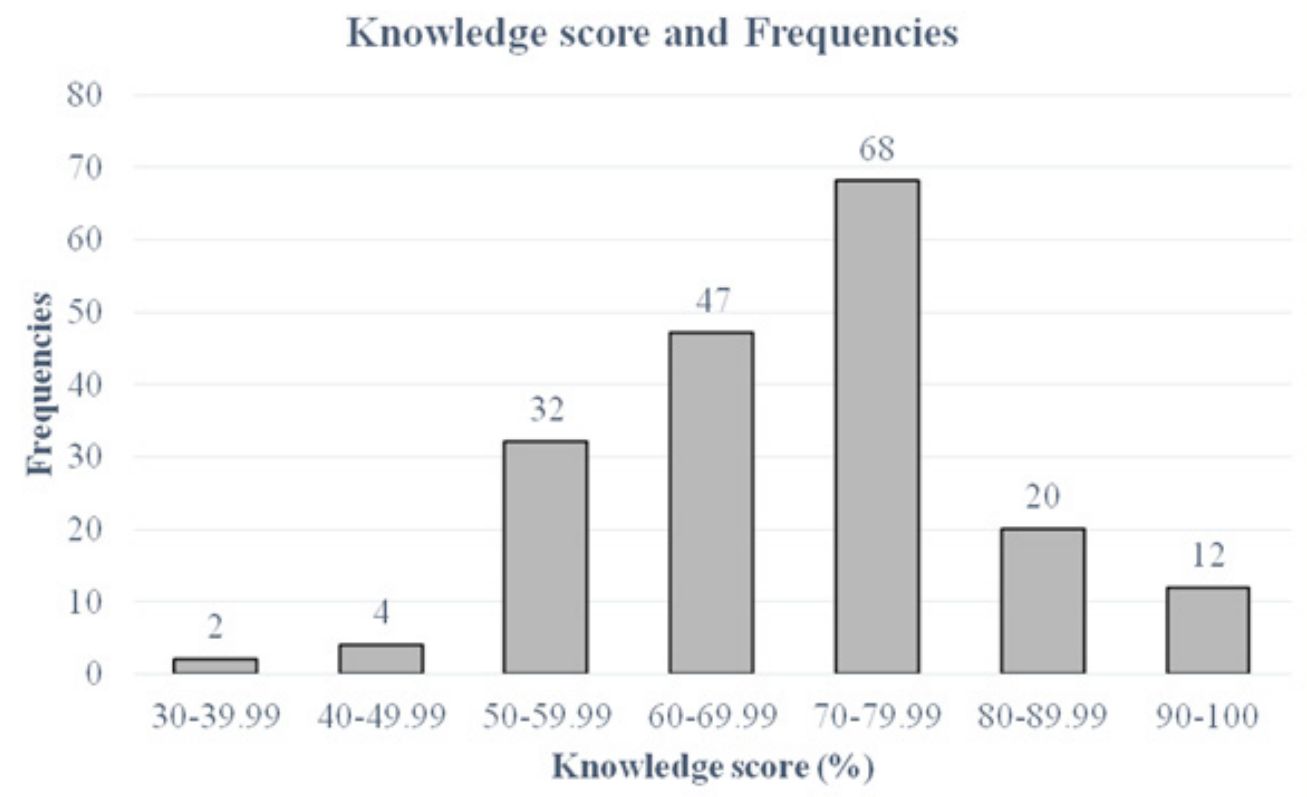

Figure 1: Graph of frequencies of knowledge score in groups.

Note: Knowledge score was scaled to percentage and grouped at intervals of $10 \%$. The $0 \%-9.99 \%, 10 \%-19.99 \%$, and $20 \%-29.99 \%$ groups are not shown because they contained no respondents. The number at the top of each bar is the number of respondents in the group. 
Table 2: Number of correct, wrong, and missing responses for knowledge items

\begin{tabular}{|c|c|c|c|}
\hline \multirow{2}{*}{ Items } & \multicolumn{3}{|c|}{$n(\%)$} \\
\hline & Correct & Wrong & Unanswered \\
\hline \multicolumn{4}{|c|}{ Section 2: Blood bag collection from blood bank and patient preparation before transfusion } \\
\hline Blood products that need cross matching & $180(97.3)$ & $4(2.2)$ & $1(0.5)$ \\
\hline Sample for cross matching & $150(81.1)$ & $31(16.8)$ & $4(2.2)$ \\
\hline Valid duration of GXM sample & $36(19.4)$ & $147(79.5)$ & $2(1.1)$ \\
\hline Bedside labelling of sample & $173(93.5)$ & $12(6.5)$ & $0(0)$ \\
\hline $\mathrm{ABO}$ and $\mathrm{RhD}$ compatibility & $83(44.9)$ & $101(54.6)$ & $1(0.5)$ \\
\hline Transporting packed red cells & $159(85.9)$ & $26(14.1)$ & $0(0)$ \\
\hline Details checked during collection of blood products & $155(83.8)$ & $29(15.7)$ & $1(0.5)$ \\
\hline \multicolumn{4}{|l|}{ Section 3: Pre-transfusion nursing responsibilities } \\
\hline Handling of blood products at wards & $105(56.8)$ & $78(42.2)$ & $2(1.1)$ \\
\hline Necessity to pre-warm blood & $84(45.4)$ & $100(54.1)$ & $1(0.5)$ \\
\hline Pre-warm method & $109(58.9)$ & $72(38.9)$ & $4(2.2)$ \\
\hline Solution co-administered with blood & $179(96.8)$ & $6(3.2)$ & $0(0)$ \\
\hline Maximum delay at wards & $128(69.2)$ & $55(29.7)$ & $2(1.1)$ \\
\hline Sequence of blood transfusion & $157(84.9)$ & $26(14.1)$ & $2(1.1)$ \\
\hline Skipping blood checking steps & $125(67.6)$ & $60(32.4)$ & $0(0)$ \\
\hline Use of filter & $175(94.6)$ & $9(4.9)$ & $1(0.5)$ \\
\hline \multicolumn{4}{|c|}{$\begin{array}{l}\text { Section 4: During and post-transfusion nursing responsibilities and management of adverse } \\
\text { reactions }\end{array}$} \\
\hline Maximum time for packed red blood cell transfusion & $173(93.5)$ & $12(6.5)$ & $0(0)$ \\
\hline Risk of exceeding recommended time & $36(19.5)$ & $147(79.5)$ & $2(1.1)$ \\
\hline Vital signs & $125(67.6)$ & $57(30.8)$ & $3(1.6)$ \\
\hline Transfusion reactions & $128(69.2)$ & $56(30.3)$ & $1(0.5)$ \\
\hline Managing transfusion reactions & $180(97.3)$ & $5(2.7)$ & $0(0)$ \\
\hline Transfusion-transmitted infections & $131(70.8)$ & $53(28.6)$ & $1(0.5)$ \\
\hline Most common cause of fatal transfusion reaction & $96(51.9)$ & $89(48.1)$ & $0(0)$ \\
\hline
\end{tabular}

\section{Knowledge of Pre-Transfusion Nursing Responsibilities}

The mean score for Section 3 was $71.75 \%$ $(\mathrm{SD}=18.63$; range $=25$ to $100 \%)$. Eighty percent of subjects $(n=149)$ were able to answer at least five out of eight items correctly. Twenty-four nurses (13.0\%) answered all items correctly. Nonetheless, more than half of the subjects $(n=100)$ had the wrong perception that every packed red cell needs to be pre-warmed before transfusion. Numerous nurses $(n=72)$ did not have proper knowledge about how to pre-warm blood units and how to handle blood products in the wards. Surprisingly, almost one-third of the subjects $(n=60)$ allowed blood checking steps to be skipped, especially when the patient was actively bleeding or unconscious (refer Table 2).

\section{Knowledge of During and Post-Transfusion Nursing Responsibilities and Management of Adverse Reactions}

The mean score $(67.14 \%, S D=16.71)$ for Section 4 was lower than that of the other sections. Eighty-three percent of subjects $(n$ $=154,83.3 \%$ ) were able to answer at least four items correctly, and only seven of them 
answered all questions correctly. Almost $80 \%$ of subjects did not know the possible consequences of a transfusion exceeding the recommended duration. Almost half of the nurses were unaware of wrong patient identification being the most common cause of a fatal transfusion reaction. In addition, nearly one-third of the subjects could not identify the correct vital signs to be monitored during a transfusion $(n=57)$ and symptoms of transfusion reactions $(n=56)$ (refer Table 2).

\section{Association of Knowledge Score with Socio-Professional Factors}

Statistically significant relationships were identified between knowledge scores and factors such as age, years of service, and availability of transfusion policy in the ward. Weak but significantly positive linear correlations were noted between age $(r=$ $0.194, p=0.004)$ and years of service $(r=$ $0.211, p<0.001)$ with knowledge score. One-way ANOVA revealed significant differences in mean knowledge scores among age groups $[\mathrm{F}(7,177)=3.118, \mathrm{p}$ $=0.004]$ and years of service $[\mathrm{F}(4,177)=$ 7.332, $p<0.001]$. A subsequent post-hoc Tukey's test revealed significantly higher mean knowledge scores among those 31 to 35 years old compared to those 21 to 25 years old. Moreover, nurses with more than 5 years of working experience scored significantly higher than the group who had just 1-5 years of clinical experience. Nurses who were aware that the blood administration policy is available in their ward performed significantly better than those who answered "Not sure" $[F(2,180)=$ 3.491, $p=0.033$.

\section{DISCUSSION}

Nurses' overall knowledge score about blood transfusion was moderate $(70.44 \%$ \pm 11.35), which implies that nurses were equipped with at least some basic knowledge about how to perform routine blood transfusion procedures. However, the mean knowledge score in this study was higher than that reported in other studies $(7-8,12)$. This could be due to the low number of knowledge items assessed in this study (22 items) compared to others (> 35 items) $(7-8,12)$, as fewer items increased the weight of each question on the $100 \%$ scale. In this study, the lowest knowledge score was recorded for the "during and post-transfusion nursing responsibilities and management of adverse reactions" section $($ mean $=67.14 \%)$. This coincided with results of another study in which the lowest score was recorded for the "during transfusion step" (12). None of the nurses in the current study scored $100 \%$, which agreed with previous studies conducted in the United Arab Emirates and Turkey (7, 13).

\section{Relationship between Knowledge Scores and Socio-Professional Factors}

Age, years of service, and availability of the transfusion policy in the ward exhibited significant relationships with overall knowledge scores. Under normal circumstances, an increased years of service corresponds to increased age, and years of service is comparable to experience level. Nurses who have worked longer have had more opportunities to attend training or courses related to blood transfusion. However, previous studies failed to show a significant relationship between experience level and knowledge score $(7,14)$, although those who received further training had significantly higher scores than those who did not $(9-10,12)$.

No significant difference in knowledge score was detected among males and females in this study, as was also reported for a study conducted in northern India (10). However, the proportion of females (96\%) outweighed that of males $(4 \%)$ in this study. Although the nursing profession is not a gender-specific job, most nurses are female. This gender imbalance was also reported in France, Arab, northern India, and Brazil (7$10,12)$. 
Higher education level usually indicates greater knowledge. However, a significant relationship between knowledge score and academic qualifications was not detected in this study. This could be because most nurses in this study $(93.5 \%)$ had a basic education (i.e., nursing certificate, diploma). Local policy states that the minimum qualification for a nursing job is having a Diploma in Nursing. A very small proportion of nurses who had worked for more than 20 years possessed a nursing certificate, as decades ago nursing certificate holders were allowed to apply for nursing jobs.

Departments and frequency of transfusion were inter-related, likely because busy wards with high workloads have increased frequency of practicing transfusion. In this study, there was no significant relationship noted between departments, frequency of transfusion and knowledge score. This could be due to the inconsistent number of nurses participated among each department.

\section{Knowledge Deficiencies}

Most nurses could not correctly state the valid duration of GXM samples. As most nurses were not based in the blood bank, this knowledge likely was unfamiliar to them. This item may have sounded ambiguous to the nurses, and wrong responses may have been due to poor comprehension.

This study revealed that $45 \%$ of nurses had not mastered the basic $\mathrm{ABO}$ and $\mathrm{RhD}$ blood group system. A similar observation was reported previously (7). Subjects could not identify the compatible blood to be transfused to a patient with a known blood group. Although local policy requires the use of group-specific blood units, there are circumstances when this rule is violated, such as when the blood bank is short of blood. Thus, nurses must have basic blood group knowledge to be able to identify errors if wrong blood units are issued.
Many nurses (45.4\%) thought that it is necessary to pre-warm blood before every transfusion. In fact, blood warming is only indicated for patients with conditions such as cold agglutinin syndrome, in neonates, and for those needing a massive transfusion. However, the term "pre-warmed blood" could have two different meanings. Some nurses could have thought that it referred to warming the blood from storage temperature to a temperature closer to room temperature $\left(20^{\circ} \mathrm{C}-24^{\circ} \mathrm{C}\right)$. However, the true definition of blood pre-warming is warming the blood unit closer to human body temperature before it is transfused to the patient to avoid hypothermia and cardiac complications. The procedure for prewarming blood involves using a calibrated blood warmer. It became a common practice in Hospital Pulau Pinang that nurses would leave a blood unit at the nurses' station for some time before transfusion, but this practice is not necessary. Moreover, this practice further delays the commencement of transfusion, which severely compromises the viability of cells and increases the risk of bacterial proliferation in blood units (4).

Almost one-third of nurses responded that final blood checking steps could be omitted if the patient is actively bleeding, unconscious, during after-hours transfusion, or if the nurse clearly knows the patient. This imposes a high chance of nurses taking a "short-cut" or skipping some important steps in ensuring that the right blood goes to the right patient. This is a bad practice that compromises the safety of blood transfusion.

The majority of nurses were not aware that the reason for the recommended duration of transfusion is to prevent proliferation of bacteria in the blood unit that may cause sepsis in the recipient. Most nurses responded that patient may develop acute transfusion reactions, which is an improbable reaction to prolonged transfusion. If nurses equip themselves with relevant knowledge, they will avoid practices that could introduce and increase the risks of bacterial contamination. 
Almost half of the nurses were not aware that most wrong blood transfusions that result in severe morbidity and mortality are caused by misidentification of patients, especially during blood sampling and final bedside checking. Consequently, this group of nurses would not pay extra attention and take the steps seriously when performing the tasks. This signifies a great risk of malpractice in the clinic.

\section{Study Limitations}

This study only explored transfusion knowledge among nurses and cannot be generalised to reflect the true transfusion practice in the wards. Some of the topics related to technical aspects of transfusion, such as calculation of flow rate and selection of filter size of the blood administration set, were not included in the questionnaire. Furthermore, this study only covered adult transfusion and excluded paediatric and neonatal transfusion. In addition, the use of the single best answer type of questions allowed respondents to guess. This study used an English questionnaire for data collection, thus nurses with poor command of English might not have been able to comprehend the questions correctly and thus may have performed poorly. Data collection was conducted using a selfadministered questionnaire, and participants might have discussed answers with coworkers and other participants despite clear instructions that discussions were prohibited. As a result, collective knowledge of small groups instead of individual knowledge may have been obtained. However, this scenario simulates the real situation in a clinical area. If a staff member is unsure of certain procedures or how to manage a patient, the first thing they likely would do is confer with their co-workers or superiors.

\section{CONCLUSION}

Blood is a unique and scarce resource that must be used cautiously to ensure its safety and efficacy. Despite extensive standards for ensuring blood safety, such as strict donor selection criteria, enhanced transfusion-transmitted infection detection, and pre-transfusion testing, few studies have examined the end-user factors affecting blood transfusion, which are keys to safe blood transfusion. In this study, the nurses questioned had moderate knowledge of the various phases of blood transfusion practice. Significant relationships between mean knowledge scores and socio-professional factors (e.g., age group, years of service, and availability of the blood administration policy in the ward) were detected. This study also revealed the lack of knowledge among nurses about basic blood group compatibilities, validity of the GXM sample, necessity of the blood warming procedure, and risk of bacterial contamination with prolonged transfusion duration.

These results highlight possible unsafe blood transfusion practices in clinical settings. Urgent action from managerial authorities is required, including providing training courses to disseminate relevant knowledge. Continuous assessment of knowledge and competencies of nurses as well as regular audits need to be conducted to ensure proper blood administration practice. In the near future, this study can be extended to involve more medical centres across Malaysia. Instead of a cross-sectional study, observational studies and simulated exercises can be conducted to provide a clearer picture of the true condition occurring in various wards (15). An interventional study can also be carried out to assess pre- and post-training knowledge levels.

\section{ACKNOWLEDGEMENTS}

This research was supported by a grant from the Advanced Medical and Dental Institute (AMDI), Universiti Sains Malaysia. We would like to extend our gratitude to Matron Nooradimahsantha binti Abdullah and Matron Kelizah bt Hamid from Hospital Pulau Pinang for their support 
and help with data collection, and Dr. Rohayu Hami, En. Nizuwan Azman, and Pn. Nurfarhana Farah Abdullah for their valuable advice about statistical and data analysis.

\section{REFERENCES}

1. McClelland DBL, editors. Handbook of transfusion medicine. 4th ed. London: TSO; 2007.

2. Singer MB, Sheckley M, Menon VG, Sundaram V, Donchev V, Voidonikolas G, Nissen NN. Can transfusions be eliminated in major abdominal surgery? Analysis of a five-year experience of blood conservation in patients undergoing pancreaticoduodenectomy. Am Surg. 2015;81(10):983-7.

3. Clevenger B, Kelleher A. Hazards of blood transfusion in adults and children. Contin Educ Anaesth Crit Care Pain. 2013;14:1128. https://doi.org/10.1093/bjaceaccp/mkt042.

4. Norfolk D. Handbook of transfusion medicine. 5th ed. Norwich:TSO;2013.

5. Bolton-Maggs PHB. Transfusion safety in 2012: main messages from the SHOT annual report for 2012. Transfus Med. 2013;23(4):217-8. https://doi.org/10.1111/ tme. 12060 .

6. Holmberg JA. Blood you can trust: global trends in transfusion safety. MLO Med Lab Obs. 2015;47(10):20.

7. Hijji B, Parahoo K, Hussein MM, Barr O. Knowledge of blood transfusion among nurses. J Clin Nus. 2013;22(17-18):253650. https://doi.org/10.1111/j.1365-2702. 2012.04078.x.

8. Hijji BM, Oweis AE, Dabbour RS. Measuring knowledge of blood transfusion:
A survey of Jordanian nurses. Am Int J Contemp Res. 2012;2:77-94.

9. Saillour-Glénisson F, Tricaud S, MathoulinPélissier S, Bouchon B, Galpérine I, Fialon P, Salmi LR. Factors associated with nurses' poor knowledge and practice of transfusion safety procedures in Aquitaine, France. Int J Qual Health Care. 2012;14(1): 25-32. https://doi.org/10.1093/intqhe/14.1.25.

10. Dubey A, Sonker A, Chaudhary RK. Evaluation of health care workers' knowledge and functioning of blood centres in north India: a questionnaire based survey. Transfus Apher Sci. 2013;49(3):565-70. https://doi. org/10.1016/j.transci.2013.09.007.

11. Smith FC, Donaldson J, Pirie L. Preregistration adult nurses' knowledge of safe transfusion practice: results of a 12 month follow-up study. Nurse Educ Pract. 2010;10(2):101-7. https://doi.org/10.1016/j. nepr.2009.04.003.

12. Tavares JL, Barichello E, De Mattia AL, Barbosa MH. Factors associated with knowledge of the nursing staff at a teaching hospital on blood transfusion. Rev Lat Am Enfermagem. 2015;23:595-602. https://doi. org/10.1590/0104-1169.0024.2593.

13. Bayraktar N, Erdil F. Blood transfusion knowledge and practice among nurses in Turkey. J Intraven Nurs. 2000;23:310-7.

14. Reza PA, Aziz SV, Ali MA, Marjan MH, Reza TM. 2009. Evaluation of knowledge of healthcare workers in hospitals of Zabol city on proper methods of blood and components transfusion. Asian J Transfus Sci. 2009;3(2):78-81. https://doi. org/10.4103/0973-6247.53878.

15. Hogg G, Pirie ES, Ker J. The use of simulated learning to promote safe blood transfusion practice. Nurse Educ Pract. 2006;6(4):214-223. https://doi.org/10.1016/ j.nepr.2006.01.004. 